\title{
Perennial Allergic Rhinitis
}

National Cancer Institute

\section{Source}

National Cancer Institute. Perennial Allergic Rhinitis. NCI Thesaurus. Code C92189.

Allergic rhinitis caused by indoor allergens and lasting year round. 